\title{
Un débat sur la vocation sacerdotale
}

La querelle Lahitton-Branchereau (1909-1912)

\section{Gérard Lefeuvre}

\section{(2) OpenEdition \\ 1 Journals}

\section{Édition électronique}

URL : http://journals.openedition.org/abpo/495

DOI : $10.4000 / a b p o .495$

ISBN : 978-2-7535-1515-4

ISSN : 2108-6443

\section{Éditeur}

Presses universitaires de Rennes

Édition imprimée

Date de publication : 30 octobre 2009

Pagination : 63-78

ISBN : 978-2-7535-1008-1

ISSN : 0399-0826

\section{Référence électronique}

Gérard Lefeuvre, "Un débat sur la vocation sacerdotale », Annales de Bretagne et des Pays de l'Ouest [En ligne], 116-3 | 2009, mis en ligne le 30 octobre 2011, consulté le 30 avril 2019. URL : http:// journals.openedition.org/abpo/495; DOI : 10.4000/abpo.495 


\title{
Un débat sur la vocation sacerdotale La querelle Lahitton-Branchereau (1909-1912)*
}

\author{
Gérard LEFEUVRE \\ Docteur en théologie, diocèse de Nantes
}

En 1896, le chanoine Louis Branchereau, prêtre de Saint-Sulpice et supérieur du grand séminaire d'Orléans, publiait un livre sur la vocation sacerdotale ${ }^{1}$. Après avoir défini la nature de la vocation, il en faisait une étude développée dans laquelle celle de "l'attrait " ou désir intérieur occupait la place essentielle ${ }^{2}$. Ce livre et la doctrine qu'il contient marquent en quelque sorte le sommet d'une période que l'on pourrait dire de possession paisible qui exprime toute la pratique des séminaires issus de ce qu'il est coutume d'appeler « l'École française " et plus exactement de l'évolution de la tradition de Saint-Sulpice, en particulier sous l'influence de M. Tronson ${ }^{3}$.

Cependant, en 1909, le chanoine Lahitton, professeur au grand séminaire d'Aire et Dax, dans un livre intitulé La Vocation sacerdotale, attaquait directement la théologie de l'attrait prônée par M. Branchereau ${ }^{4}$. Il y affirmait que « la vocation sacerdotale n'existe dans un sujet qu'après qu'elle y a été apportée du dehors par l'appel des légitimes pasteurs de l'Église. Vocari autem a Deo dicuntur qui a legitimis Ecclesiae ministris vocantur ». Plus tard, dans une édition plus élaborée, il reprenait intégralement ses premières affirmations : "La vocation vient de Dieu par l'évêque, pas d'appel divin à chercher dans le sujet [...] La vocation en actes, ou l'appel divin, c'est l'évêque qui la défère, qui la donne, qui la crée ${ }^{5}$. " Cependant, il n'attaquait pas directement les fondements théoriques de la théologie de l'attrait, se bornant à dire " que la théorie de la vocation devient une des plus fuyan-

* Cet article est issu d'une communication orale présentée en avril 2007 à l'université du Maine lors d'une journée d'étude organisée par Hervé Guillemain et consacrée aux vocations religieuses et laïques.

1. Branchereau Louis, De la Vocation sacerdotale, Paris, Vic et Amat, 1896.

2. Cf. chapitres 8 à 12 .

3. KRUMENACKER, Yves L'École française de spiritualité. Des mystiques, des fondateurs, des courants et leurs interprètes, Cerf-Histoire, 1998.

4. LAHITTON Joseph, La Vocation sacerdotale, traité théorique et pratique à l'usage des séminaires et des recruteurs de prêtres, Paris, Lethielleux, 1909.

5. LAHITTON Joseph, La Vocation sacerdotale..., op. cit., p. 1. 
tes de la théologie catholique " et il citait des expressions recueillies dans des livres de piété : "Sa vocation est en danger - Il a perdu sa vocation - Il n'avait pas une bonne vocation - Le sacerdoce est la plus belle des vocations - Ce milieu est fécond en vocations ${ }^{6}$."

Ce livre déclancha de vives controverses dans les séminaires et tous les milieux attentifs au discernement des vocations. Ce fut l'origine de la " querelle ". D'après une confidence faite à l'auteur par un de ses anciens élèves, Mgr Cazaux, évêque de Luçon, la réflexion du chanoine Lahitton fut provoquée par le problème posé par des séminaristes, jugés modernistes par leurs directeurs du grand séminaire de Dax, mais qui prétendaient avoir le droit d'être ordonnés à cause de leur certitude d'avoir " la vocation divine ", c'est-à-dire à cause de leur certitude intérieure d'être appelés par Dieu au sacerdoce.

Pour comprendre l'enjeu des controverses ainsi suscitées, il faut brièvement rappeler d'abord le concept de vocation puis comment on en est venu, depuis la Réforme, à celui de vocation sacerdotale ${ }^{7}$.

Le terme vocation vient de vocatio issu de la forme latine vocare: " appeler ". Le terme français vocation a originellement un sens spécifiquement chrétien. Ce n'est que secondairement qu'il a pris un sens profane, comme le montre l'exemple de quelques dictionnaires. Ainsi le Littré ou le Dictionnaire de la langue française de Robert, qui distingue un sens biblique "Appel de Dieu touchant une personne, un peuple, afin qu'il vienne à lui " et un sens religieux "Mouvement intérieur par lequel on se sent appelé par Dieu ", puis "Inclination, penchant pour une profession, un état ". Le Dictionnaire historique de la langue française précise : terme biblique. "Par extension de la valeur religieuse, le mot désigne la destination d'une personne et l'inclination qu'éprouve quelqu'un pour une profession, un état ". $\mathrm{Au} \mathrm{xx}^{\mathrm{e}}$ siècle, le mot a pris le sens large de rôle auquel un peuple, un pays paraît appelé et s'emploie dans la locution " avoir vocation à ".

L'“ appel à " renvoie immédiatement à trois réalités : celui qui appelle; celui qui est appelé; le but de l'appel. Dieu appelle quelqu'un pour une mission. Selon les circonstances et les époques, l'accent sera mis sur l'un ou l'autre des aspects, et chacun d'eux pose des problèmes mais il est essentiel de prendre le point de départ dans la révélation : la vocation au sens propre est un mystère lié à une initiative de Dieu.

\section{La vocation sacerdotale}

La réalité spirituelle exprimée par le terme "vocation " a fait l'objet, surtout depuis la fin du XIX ${ }^{\mathrm{e}}$ siècle de réflexions et d'études multiples parmi lesquelles la « querelle Lahitton-Branchereau » a tenu une place importante.

6. Ibidem, p. 122-123.

7. Pour un développement plus important, on peut consulter LEFEUVRE Gérard, $L a$ Vocation sacerdotale dans le second Concile du Vatican, Paris, Téqui, 1978 et dans le dictionnaire Catholicisme, tome XV, fascicule 74, article Vocation avec bibliographies. 
Elle peut nous permettre de situer quelques aspects de ce que l'on a appelé la vocation sacerdotale, expression ecclésiale de ce que l'on a nommé la vocation divine.

Pour situer ce concept relativement récent, il convient de remonter rapidement dans l'histoire jusqu'à la Réforme qui, dans ce domaine aussi, a joué un rôle tout particulier. Pour Luther, son expérience religieuse personnelle sur fond de solitude et sa réflexion théologique sur l'épître aux Romains l'ont conduit peu à peu à rejeter, au nom de la justification par la foi, les oeuvres et la médiation de l'Église, d'où sa conception de la vocation. Pour beaucoup, en effet, la définition de la vie monastique comme état de perfection avait comme absorbé en elle la notion de vocation; l'état monastique était source de difficultés, surtout à cause d'une affirmation sous-jacente à divers courants de pensée selon lesquels les obligations liées par vœux à l'état religieux avaient par elles-mêmes une valeur spirituelle et donnaient une certitude plus grande à l'égard du salut. Luther voit là une opposition absolue au principe même de la justification. Ceci entraîne, audelà de sa critique virulente de la décadence monastique - lieu commun de l'époque - un rejet théorique de la vocation religieuse, à la fois comme oeuvre méritoire mais aussi dans sa réalité même de vocation c'est-à-dire d'appel particulier à la perfection. Au-delà de la polémique, Luther reprend l'affirmation d'une vocation générale pour tous les baptisés : chaque état constitue proprement une vocation au sein de laquelle il s'agit de répondre personnellement à l'appel de Dieu par la foi. La même référence au sujet seul et à la sola gratia se retrouve quand il s'agit du sacerdoce. Être croyant, c'est être prêtre du peuple de Dieu; le prêtre n'est rien d'autre qu'un croyant et le pasteur auquel la communauté confie le ministère de la prédication n'est pas constitué par l'ordination dans un état distinct de celui du laïc. Il ne peut donc y avoir de vocation sacerdotale.

Calvin a suivi pour sa part les orientations fondamentales de la pensée de Luther mais avec des différences qu'il faut relever ici. Par rapport à la vocation : la vocation générale est promesse et exigence à la lumière de la Loi de Dieu, révélée dans les Écritures; la vocation particulière sera légitime si elle est conforme à la Loi ; autrement elle sera apparente comme celle des moines qui vivent selon la superstition : celle-ci s'appuie sur une fausse image de Dieu en prétendant que certaines situations sociales sont plus aptes à le servir que d'autres; la vocation ne détermine pas de structure ecclésiale permettant un état de vie meilleur, mais elle consiste à se laisser réformer par l'Esprit Saint. Par rapport surtout à la prédestination : Calvin la met en relief et voit en elle, à travers la variété des situations concrètes un secret admirable du jugement de Dieu car elle souligne en fait la réalité de la sola gratia : " nous appelons prédestination le conseil éternel de Dieu, par lequel il a déterminé ce qu'il voulait faire de chaque homme. Car il ne les crée pas tous en pareille condition, mais ordonne les uns à la vie éternelle, les autres à l'éternelle damnation... " Et cette dualité ne contredit pas la vocation universelle, car la proclamation de l'Évangile " pour les uns 
devient vocation efficace... chez les autres, elle endurcit, aveugle, éloigne de Dieu; ceux qui étaient destinés au salut sont gracieusement sauvés, tandis que les réprouvés attirent sur eux leur juste perdition ". Il ne peut en effet y avoir de vraie coopération humaine à la grâce mais seulement correspondance, et le salut ne peut être acquis par des mérites ${ }^{8}$. Par rapport au ministère enfin, Calvin est le premier à esquisser des critères de vocation. Il est nécessaire, en effet, d'organiser la vie spirituelle de la communauté. S'il rejette, comme Luther, toute idée de l'ordre comme sacrement, il distingue deux aspects dans l'appel à la charge pastorale : la vocation intérieure, qui est le désir d'accéder au ministère, et la vocation extérieure, par laquelle la communauté confirme l'aspiration du candidat.

\section{La question de la vocation divine}

Au début du XVII ${ }^{\mathrm{e}}$ siècle, le mot vocation signifie encore la mission qui incombe du fait de l'ordination. Bérulle l'emploie en ce sens dans ses Discours de controverse : les prêtres catholiques ont une véritable vocation, tandis que les pasteurs réformés sont sans vocation faute d'avoir été ordonnés par un évêque. Mais, antérieurement à l'ordination, n'y a-t-il pas une vocation divine qui y dispose certains plutôt que d'autres? Les discussions autour de la Réforme protestante avaient contribué à remettre en lumière et à élargir l'emploi du terme vocation (alors pratiquement réservé à la vocation religieuse) au sens d'appel intérieur venant de Dieu. Quand l'expression vocation intérieure entrera dans le langage catholique, elle exprimera le recours, déjà traditionnel, au critère de l'expérience interne tel qu'il est enseigné par les Exercices de Saint Ignace. Saint Ignace appelle vocation divine l'appel adressé personnellement à chaque homme. Le Christ appelle " tous les hommes". Il y a donc non seulement une vocation générale au salut, mais une vocation correspondant à la grâce personnellement donnée à chacun. Ceci fait partie du mystère même de Dieu et de son action dans le monde. Le " Principe et fondement " des Exercices ne peut pas se comprendre sans cette affirmation d'une Providence éternelle attentive à chacun. De cette Providence émane l'appel divin, la vocation divine que chacun doit s'efforcer de discerner et de rejoindre par une bonne "élection ". Mais on voit la difficulté : comment faire le lien entre ce que Dieu veut et un choix concret? Saint Ignace demeure délibérément au niveau du choix personnel de chacun dans sa propre situation.

Cette conception marque l'importance du discernement. Le XVII ${ }^{\mathrm{e}}$ siècle est l'époque des directeurs de conscience et la direction restera la marque distinctive des institutions consacrées à la formation des futurs prêtres.

Le Catéchisme du Concile de Trente quant à lui reprend la doctrine du Concile sur le sacrement de l'Ordre en insistant sur trois aspects : la gran-

8. Cité par BüHLER, Paul, Prédestination et Providence dans Encyclopédie du Protestantisme, Paris-Genève, 1995, p. 1191. 
deur du prêtre, liée au pouvoir eucharistique conféré par le sacrement; la mission du prêtre, dérivée de celle du Christ; l'appel de l'Église :

"Dans tous les temps les prêtres ont joui des plus grands honneurs; mais les prêtres du Nouveau Testament l'emportent de beaucoup sur tous les autres en dignité. Le pouvoir qu'ils ont de consacrer et d'offrir le corps et le sang de Notre-Seigneur, et celui de remettre les péchés, surpasse toute intelligence humaine. On ne peut rien trouver sur la terre qui y soit comparable. Enfin comme Notre-Seigneur avait été envoyé par son Père, et comme les apôtres et les disciples furent envoyés par Jésus-Christ dans tout l'univers; ainsi tous les jours les prêtres sont envoyés avec le même pouvoir, pour travailler à la perfection des saints, aux fonctions de leur ministère, à l'édification du corps de Jésus-Christ. Ainsi il ne faut confier témérairement à personne les fonctions d'un si grand ministère sinon à ceux-là seulement qui sont en état de le remplir par la sainteté de leur vie, par leur science, leur foi et leur prudence. Que personne donc ne s'attribue à lui-même cet honneur, s'il n'y est pas appelé par Dieu comme Aaron (Heb 5/4) : c'est-à-dire s'il n'y a pas été appelé par les ministres légitimes de l’Église ${ }^{9}$."

\section{Un idéal sacerdotal : Bérulle et la grandeur du sacerdoce}

Le Concile de Trente avait réaffirmé fortement la réalité sacramentelle de l'ordre, le " caractère sacerdotal» lié à l'ordination faite par l'Évêque ainsi que le lien étroit de la mission du prêtre avec la célébration de la messe, réitération de l'unique sacrifice du Christ. Après avoir rappelé l'origine divine de la mission, Bérulle démontre comment, prolongeant dans le temps la mission du Christ, celle du prêtre s'inscrit dans le dessein divin de salut et exige donc une sainteté correspondant à la grandeur de cette mission qui est la restauration du lien entre l'homme et Dieu. Tel est le fondement de cette exaltation du sacerdoce qui sera la marque de ce que l'on a appelé l'École française ${ }^{10}$.

La réalité était loin de cet idéal. M. Olier, curé de Saint-Sulpice, en fit non seulement l'expérience, comme beaucoup d'autres, mais son zèle pour la réforme du clergé fut à l'origine d'une des institutions qui fit le plus pour la mettre en pratique. Le trop grand nombre de prêtres sans idéal sacerdotal restera longtemps une plaie vive de l'Église. Il suffit de se référer ici aussi au Catéchisme du Concile de Trente:

"Les uns n'embrassent l'état ecclésiastique que pour avoir de quoi subsister; ils ne cherchent que le gain en s'y engageant, comme font la plupart de ceux qui embrassent les métiers les plus bas et les plus vils... D'autres sont conduits au sacerdoce par l'ambition et le désir des honneurs. Il en est enfin qui ne recherchent les ordres que pour s'enrichir; et ce qui en est la preuve, c'est qu'ils ne pensent point à recevoir aucun des ordres sacrés, tant qu'on ne leur offre point quelque riche bénéfice. Ce sont ceux-là que Notre-

9. Catéchisme du Concile de Trente, Paris, 1830, Ch. Xxvı : Du sacrement de l'Ordre, t. 2, p. 19-20.

10. KRumenacker, Yves, L'École française de spiritualité..., op. cit., p. 199. 
Seigneur appelle des mercenaires, et dont Ezéchiel disait qu'ils se paissent eux-mêmes et non leurs brebis. La honte et le dérèglement de leur conduite a déshonoré l'état ecclésiastique aux yeux des fidèles, qui le regardent presque maintenant comme l'état le plus vil et le plus méprisable. Aussi ne tirent-ils point d'autre fruit de leur sacerdoce, que celui que tira Judas de son apostolat, c'est-à-dire leur perte éternelle ${ }^{11}$. "

Comment réformer le clergé pour avoir des prêtres vraiment tout donnés à la mission? On peut, certes, chercher à leur faire prendre conscience des obligations de leur état, mais ne faudrait-il pas d'abord chercher à empêcher d'accéder au sacerdoce ceux qui se présentent à l'ordination dans un but purement séculier? Ne faudrait-il pas chercher à n'admettre que ceux qui ont un certain idéal sacerdotal? Ainsi, non seulement on perçoit la nécessité d'une formation des futurs prêtres, mais on sent aussi le besoin de déterminer des critères valables pour ce choix. Le principe de la réforme fut la conviction qu'il fallait changer les motivations de l'accès au sacerdoce; seules des raisons spirituelles pouvaient justifier le désir d'accéder aux ordres, mais qui pouvait en juger? La réponse à cette question s'éclaira peu à peu, par l'union de la réflexion et de la pratique, grâce à l'élaboration d'une conception renouvelée de la vocation et par les initiatives qui conduisirent à la fondation des séminaires.

Dans le même sens, Saint-Cyran, avait repris l'idée de " vocation divine " et cherché à en préciser les marques, de manière à détourner du sacerdoce ceux en qui elle fait défaut, et il insistait fortement sur la correspondance nécessaire entre la vocation intérieure signifiée immédiatement par Dieu et la vocation extérieure qui est l'appel par l'évêque. De son côté, M. Olier manifestait un souci analogue. Le curé de Saint-Sulpice avait eu d'abord pour but de préparer les ordinands à la réception du sacrement de l'ordre; ensuite, dans le séminaire tel qu'il s'établit à Saint-Sulpice et qu'il se répandit en France à la demande des évêques, l'objectif principal fut de discerner, grâce à une formation spirituelle et intellectuelle adéquate, l'authenticité de la "vocation sacerdotale". Le discernement de la vocation put ainsi devenir, autant que la formation, l'objectif des séminaires. Il faut chercher ceux qui ont vraiment une vocation divine. La réflexion sur la signification exacte de ce terme fut à l'origine de toute une pastorale fondée sur le désir du service de l'Église dans le sacerdoce.

\section{La théologie de l'attrait}

La considération de l'attrait dans la recherche de la vocation se situe dans la ligne subjective qui marque l'époque moderne. L'attrait apparaît donc d'abord, en tant que désir de vivre dans l'état sacerdotal, comme une marque de vocation sérieuse. La vocation sacerdotale est nettement concue comme une réalité spirituelle spécifique, la plus haute de toutes les vocations. Il faut donc discerner les "signes de vocation ». Telle fut la

11. Catéchisme du Concile de Trente, op. cit., p. 21. 
pensée de M. Olier et de ce qu'on a appelé l'École de Saint-Sulpice. Le Traité des Saints Ordres - publié en 1675 sous le nom de M. Olier - définissait ainsi l'intention d'embrasser l'état ecclésiastique : «Un mouvement de Dieu qui porte toute l'âme et qui l'incline à cette divine profession, non par sentiment, ni par saillie ou par différentes reprises, mais par empire, par état et consistance immuable en son fond. " Cette conception de la vocation intérieure marque toute la théologie de la vocation dans la grande tradition des séminaires du XVIII ${ }^{\mathrm{e}}$ et du XIX ${ }^{\mathrm{e}}$ siècles.

Après la Révolution, la restauration des séminaires se fit en France dans la ligne du XVIII ${ }^{\mathrm{e}}$ siècle et sous influence de la Compagnie de Saint-Sulpice; la théologie de l'attrait devint peu à peu prépondérante. L'insistance sur la notion d'un attrait perçu à l'intime de l'âme fit que ce dernier finit par être considéré comme le signe principal et presque unique de l'appel divin. Comme nous l'avons vu en commençant, M. Louis Branchereau, de SaintSulpice, supérieur du grand séminaire d'Orléans, est le théoricien le plus célèbre de la tendance à voir dans l'attrait le signe principal et presque unique de l'appel divin. S'y ajoutait la prédominance donnée à l'autorité du directeur, dont le rôle est de reconnaître au for interne la réalité de l'appel, par rapport à l'autorité de l'évêque qui appelle aux ordres. L'attrait, longuement étudié, devient le critère déterminant, et il est sanctionné par le directeur dont la décision "sera la sentence définitive, le jugement en dernier ressort ${ }^{12}$ ", et l'auteur conclut que là où il y aurait des qualités précieuses et une estime du sacerdoce, mais non point du goût ou de la sympathie pour lui, il faut généralement se prononcer contre la vocation.

Le Concile de Trente, dans le contexte social de l'époque, avait prescrit d'éduquer dans les séminaires des enfants à partir de 12 ans. La restauration des séminaires, au XIX ${ }^{\mathrm{e}}$ siècle, s'accompagna de la fondation des écoles presbytérales, des petits séminaires et de maisons de formation analogues dans les ordres religieux, congrégations etc. Dans ce contexte, la vocation de l'enfant Samuel devint, et pour longtemps, un des thèmes préférés de la pastorale consacrée au recrutement des enfants. Si on tient compte de l'état du clergé au sortir de la Révolution, le redressement opéré dans les diocèses avec tant d'efforts et l'extraordinaire développement missionnaire suscité par les nombreuses congrégations masculines et féminines fondées au cours de ce siècle suffisent à montrer la valeur de la " théologie de l'attrait " dans la mesure où le désir de répondre à un appel de Dieu, de le servir ainsi que l'Église est bien à la source de toute vocation authentique sacerdotale ou religieuse. Sa mise au premier plan n'était cependant pas sans danger.

\section{La vocation-prédestination}

Comment en effet l'idée de prédestination a-t-elle pu contaminer la théologie de la vocation? Il faut revenir $\mathrm{au} \mathrm{XVII}^{\mathrm{e}}$ siècle. Les discussions sur la

12. BRANCHEREAU Louis, De la Vocation sacerdotale, op. cit., p. 268-269. 
prédestination - liées elles-mêmes aux controverses sur la grâce - étaient déjà depuis longtemps dans les perspectives du temps. À partir du moment où, de manière plus ou moins nominaliste, on se met à penser la prédestination sur le mode logique, cette notion devait inévitablement interférer avec celle de vocation divine, dès lors que cette dernière était pensée elle aussi comme un en-soi, dans l'éternité divine, au lieu d'être considérée à la manière ignatienne comme le terme d'une recherche existentielle.

Que Dieu prédestine chacun à une vocation (un état) était une manière de penser assez répandue chez les catholiques aussi bien que chez les réformés. Une première interférence se produisit lorsque, dans l'argumentation destinée à écarter du sacerdoce les candidats qui le demandent pour des motifs mondains, on vit dans ces démarches intéressées comme une marque de non-prédestination. C'est ce qu'on peut voir par exemple dans quelques textes de Bourdaloue et de Massillon. Après avoir énergiquement condamné les parents qui engagent par intérêt personnel leurs enfants dans le clergé ou la vie religieuse, Bourdaloue ajoute :

"Écoutez une des plus grandes vérités de la morale chrétienne. C'est que rien n'a tant de rapport au salut que la vocation à un état, et que souvent c'est à l'état qu'est attachée toute l'affaire du salut : comment cela? Parce que l'état est la voie par où Dieu veut nous conduire au salut; parce que les moyens de salut que Dieu a résolu de nous donner ne nous ont été destinés que conformément à l'état; parce que, hors de l'état, la Providence de Dieu n'est plus engagée à nous soutenir par ces grâces spéciales qui assurent le salut, et sans lesquelles il est d'une extrême difficulté de parvenir à cet heureux terme ${ }^{13}$."

\section{Et encore dans l'instruction sur le choix d'un état de vie:}

" Il n'y a rien dont le salut dépende davantage que de bien choisir l'état où l'on doit vivre, parce qu'il est certain que presque tous les péchés des hommes viennent de l'engagement de leur état [...]. Voilà ce qui s'appelle le secret de la prédestination, lequel roule principalement sur le choix de l'état ${ }^{14}$."

De même Massillon vers 1701, sur la vocation :

"La sainteté est la vocation générale de tous les fidèles [...]. Néanmoins la voie pour arriver à ce terme heureux n'est pas la même pour tous les hommes [...] si l'élection des justes n'est que la préparation éternelle des moyens qui doivent infailliblement les délivrer, le choix d'un état de vie étant sans doute le principal, il a dû être renfermé dans cette volonté miséricordieuse, qui leur a préparé des voies sûres de salut; et d'un autre côté, la destinée des méchants, devant servir aussi, dans les desseins de Dieu, par mille rapports secrets au salut des justes, elle a dû entrer dans le plan éternel de leur justification, et n'être pas moins arrêtée, dès le commencement, que la condition même des élus. Il demeure donc établi qu'avant que nous fussions nés, le Seigneur avait tracé à chacun de nous le plan de nos destinées, et, pour ainsi

13. BouRDALOUE, Louis, S.J., EEuvres complètes, Versailles, 1812, tome 5, p. 8.

14. Ibidem, tome 9, p. 292. 
dire, le chemin de notre éternité; et que, parmi cette multiplicité de voies qui forment les diverses conditions de la société, il n'en est qu'une qui soit la nôtre, et par où Dieu ait voulu nous conduire au salut ${ }^{15}$."

Une seconde interférence entre l'idée de vocation divine et celle de prédestination concerne l'identification qui se fit peu à peu au cours du siècle dernier entre l'idée de vocation divine au sens subjectif - le désir de servir Dieu et l'Église dans le sacerdoce, par opposition à la recherche du sacerdoce par intérêt - et celle de prédestination au sacerdoce. Tout était question d'équilibre et de nuances : si pour la plupart l'attrait pour le sacerdoce chez des enfants était considéré comme un point de départ que les années devaient mûrir et éprouver, l'idée de vocation-prédestination servit chez certains de soubassement à une théologie de l'attrait conçu comme manifestant concrètement un appel de Dieu existant de toute éternité. C'est essentiellement au sujet du discernement des vocations d'enfants que l'on cherche des signes positifs de prédestination. Il semblait impensable, en effet, que la Sagesse de Dieu, qui de toute éternité, prédestine un homme au sacerdoce ne le manifeste pas extérieurement dès l'enfance par des signes de prédestination qu'il s'agit dès lors de discerner et de protéger. Cette manière de considérer le fondement de la vocation fut semble-t-il comme un arrière-fond qui ne posait pas alors de problème particulier. C'est ainsi que le Père Giraud S.M. intitulait la troisième partie de son livre sur la vocation Du développement de la grâce sacerdotale depuis l'élection éternelle jusqu'à la prêtrise et à l'épiscopat. Dans le chapitre deux - Les signes de l'élection éternelle. L'enfant sacerdotal -, il parle ainsi de la pastorale des vocations : "Nous devons beaucoup prier pour qu'il plaise à Celui qui "connaît les cœurs de tous" et qui depuis l'éternité "a fait son choix" de nous faire discerner quel est sur vingt, sur dix, sur deux peut-être l'enfant qui est l'objet de ce choix. " Faute d'un tel discernement « tel enfant... prédestiné à la plus éminente des vocations, demeure misérablement dans les voies communes et pleines de périls de la vie du siècle... Dieu est privé d'une grande gloire, l'Église d'un soutien, les âmes d'un père; et l'enfant sacerdotal, inconnu en ce moment, mais réellement présent dans cette multitude qui se meut sous nos yeux, sera à jamais hors du plan primitif de Dieu ${ }^{16}$... " À la même époque, M. Branchereau faisait lui-même de la vocation-prédestination le fondement de sa théologie de l'attrait:

"Au point de vue strictement théologique... [le terme vocation] exprime la manifestation et l'intimation dans le temps, d'un décret porté de toute éternité, par lequel Dieu, d'une part, assigne à une créature la place qu'elle doit occuper dans le monde, le rôle qu'elle doit y remplir et la fin à laquelle elle doit tendre pour concourir à l'ordre universel; de l'autre lui assurer les aptitudes et les secours dont elle aura besoin pour atteindre ce but ${ }^{17}$. "

15. MassiLlon, $\mathrm{M}^{\mathrm{gr}} \mathrm{Jean}-B a p t i s t e$, évêque de Clermont, Euvres complètes, Bar-le-Duc, 1865 , tome 1, p. 504-505.

16. Prêtre et hostie, $1891,5^{\mathrm{e}}$ éd., 1924.

17. BRANCHEREAU, Louis, La Vocation sacerdotale, op. cit., p. 14-15. 
Au plan concret, l'auteur s'exprime en termes quasi juridiques :

"Ce décret éternel est, pour l'être libre, une loi. Il faut pour qu'elle obtienne son effet, qu'elle soit promulguée et intimée à ceux qu'elle oblige. " La vocation ainsi entendue présente trois caractères : " $1^{\circ}$ elle est divine dans son principe $[\ldots] 2^{\circ}$ elle est personnelle $[\ldots] 3^{\circ}$ elle est impérative, c'est-àdire qu'elle implique, pour les êtres dont elle fixe la destinée, la nécessité ou l'obligation ${ }^{18}$."

Le 20 juin 1912, une commission cardinalice, nommée par le pape pour juger du débat Lahitton-Branchereau, estimait le livre du chanoine Lahitton :

"particulièrement digne d'éloges en ce qu'il établit les trois points suivants : $1^{\circ}$ nul n'a jamais aucun droit à l'ordination, antérieurement au libre choix de l'évêque; $2^{\circ}$ la condition qu'il faut examiner du côté de l'ordinand, et qu'on appelle vocation sacerdotale, ne consiste nullement, du moins nécessairement, et en règle ordinaire, dans un certain attrait intérieur du sujet ou dans une invite du Saint-Esprit, à embrasser l'état ecclésiastique; $3^{\circ}$ mais, au contraire, pour que l'ordinand soit régulièrement appelé par l'évêque, rien de plus n'est exigé de lui que l'intention droite unie à l'idonéité; celle-ci consiste en de telles qualités de nature et de grâce; elle s'affirme par une probité de vie et une mesure de science telles, qu'on en puisse concevoir l'espérance fondée que le sujet sera capable de remplir convenablement les fonctions du sacerdoce et d'en garder saintement les obligations. "

Ainsi, le premier point correspond à la préoccupation majeure du chanoine Lahitton et à l'intuition qui a orienté sa position, mais la question n'était pas entièrement éclaircie par cette déclaration cardinalice. Par la suite, divers documents que nous allons indiquer brièvement essaieront de préciser les différents points de vue.

Dès 1917, le Code de droit canonique donnait une première indication en parlant, au sujet des enfants à admettre dans les séminaires, de " signes de vocation sacerdotale " et surtout de " germes de l'appel divin ». Trois autres textes achèvent de situer la signification de cette réintroduction de l'attrait dans une conception plus complète et mieux équilibrée de la vocation.

En 1930, une instruction de la Congrégation des sacrements prescrivait à tous les ordinands candidats aux ordres majeurs de signer une déclaration attestant de la complète liberté de leur démarche : "Au moment de recevoir l'ordination et après y avoir réfléchi en présence de Dieu, j'atteste sous serment que je demande à recevoir cet ordre de mon plein gré; sans y être poussé par une quelconque pression, violence ou crainte, mais que je le veux d'une liberté pleine et entière, ayant éprouvé avec certitude que j'étais vraiment appelé par Dieu. "

En 1935, le pape Pie XI, dans son encyclique Ad catholici sacerdotii fastigium consacrée au sacerdoce, précisait quelque peu la signification de cette conscience subjective de répondre à un appel divin, qu'on indique couram-

18. Ibidem, p. 17-19. 
ment du nom de « vocation " et il en affirme l'existence réelle : l'homme peut prendre conscience, intérieurement, d'un appel qui correspond vraiment à un dessein divin sur lui.

En 1956, enfin, la Constitution apostolique Sedes Sapientiae du pape Pie XII chercha à concilier la vocation canonique, réalisée par l'appel de l'évêque, et la vocation intérieure, grâce divine qui pousse à vouloir le sacerdoce et à s'y préparer.

" Le fondement de toute vie religieuse, sacerdotale et apostolique, c'est-àdire la vocation divine, comporte en quelque sorte deux éléments essentiels, l'un divin, l'autre ecclésiastique. En ce qui concerne le premier, l'appel de Dieu, nous dirons qu'il est à ce point nécessaire pour entrer dans l'état religieux ou sacerdotal que, s'il faisait défaut, on doit affirmer que le fondement même sur lequel s'appuie tout l'édifice fait défaut. Celui en effet que Dieu n'appelle pas, sa grâce ne le conduit ni ne l'assiste... Pour en venir ensuite au second élément de la vocation religieuse et sacerdotale, le catéchisme romain enseigne que "sont dits appelés par Dieu ceux qui sont appelés par les ministres légitimes de l'Église." Cela est loin d'être en contradiction avec ce que nous avons dit de l'appel de Dieu, mais s'y trouve plutôt étroitement lié. La vocation divine à l'état religieux et clérical - puisqu'elle destine quelqu'un à mener publiquement une vie de sanctification et à exercer un ministère hiérarchique dans l'Église, société visible et hiérarchique - doit recevoir confirmation, acceptation et direction autorisées de la part des supérieurs, également hiérarchiques, à qui a été confiée par Dieu le gouvernement de l'Église."

Ces différentes interventions forment le contexte théologique dans lequel se situera le travail du Concile Vatican II au sujet de la vocation sacerdotale. On remarquera cependant que rien jusque-là n'a fondamentalement changé dans la problématique. Comme s'il ne s'était rien passé depuis le renouveau des petits et grands séminaires au dix-neuvième siècle jusqu'à l'époque du second Concile du Vatican. On peut penser d'ailleurs qu'il y a encore de nombreux prêtres qui ont entendu sans sourciller dans leurs petits séminaires des lectures spirituelles dans la plus pure tradition de la vocation-prédestination, mais aussi que les conseils avisés des professeurs auxquels ils s'adressaient en confession remettaient sans drame les choses en place, à supposer qu'ils aient bien écouté et compris ce qui leur avait été dit auparavant.

\section{Excursus}

Puisque l'allusion ci-dessus n'est pas sans rappeler quelques souvenirs personnels, on me pardonnera peut-être d'en évoquer deux autres qui peuvent illustrer des aspects de ce qui fut sans doute, de manière analogue, le cheminement de beaucoup. Dans les années 1939-1940, les petits choristes du couvent des franciscains de Saint-Nazaire n'étaient pas toujours d'une sagesse exemplaire. Une chose pourtant m'a particulièrement frappé puisque je ne l'ai pas oubliée. Selon les règles liturgiques, au Salut du Saint- 
Sacrement, les deux choristes étaient à genoux sur le premier degré de l'autel de chaque côté du célébrant dont ils relevaient légèrement les côtés de la chape afin qu'il puisse manier plus facilement l'encensoir. Or il y avait un vieux père (au moins à nos yeux, mais il devait être quand même assez âgé), le père R. qui avait une telle expression indéfinissable lorsqu'il encensait le Saint-Sacrement que j'en demeurais frappé et je me souviens d'avoir pensé une fois : "Comme je voudrais bien faire comme lui un jour! »

Une douzaine d'années plus tard, alors que j'achevais mon service militaire comme instructeur à l'école des apprentis mécaniciens de l'Armée de l'Air de Rochefort et Saintes, l'ambiance était fort sympathique et un officier, le capitaine X., m'encourageait un jour à rester dans l'armée : « On manque d'ingénieurs et vous pouvez faire une belle carrière; pourquoi vouloir rentrer au grand séminaire? " Je crois lui avoir répondu en substance à peu près ceci : "C'est vrai ce que vous dites, mais la seule chose dont je suis sûr, c'est que, si je ne rentre pas au séminaire, je ferai sans doute la plus grosse sottise de ma vie! " Comme on le voit, j'étais bien loin psychologiquement des théories, et bien incapable, comme tout le monde d'ailleurs, de prévoir les directions successives que j'allais prendre, et aller même jusqu'à choisir ces mêmes théories sur la vocation comme sujet de thèse, ce qui peut donner à réfléchir aux psychologues.

Quoi qu'il en soit, il me reste simplement à dire en terminant quelques mots de l'enseignement du Second Concile du Vatican à ce sujet.

\section{L'enseignement du Concile Vatican II}

Des différentes phases préparatoires à la tenue du Concile, nous pouvons retenir seulement un texte significatif : en réponse à la demande d'une définition de la vocation sacerdotale, un projet de la Congrégation des Séminaires et Universités voit dans celle-ci " une réalité spirituelle dépendant d'une action particulière de Dieu, action dont l'existence est certaine si on considère, conformément à la doctrine courante, qu'elle découle nécessairement de la grandeur de l'état sacerdotal ". On perçoit déjà deux problèmes : comment exprimer en termes abstraits ce qui se situe essentiellement au niveau de l'expérience; et comment établir la distinction entre la vocation divine et la vocation chrétienne autrement qu'en termes de supériorité et d'infériorité? Conception en effet très répandue, comme en témoignent par exemple ces lignes du P. Sempé : le sacerdoce est « une participation à l'œuvre rédemptrice du Christ. Or le simple titre de chrétien ne donne évidemment pas droit à un pareil honneur. Le chrétien, comme tel, ne peut prétendre qu'aux grâces qui lui sont personnellement nécessaires pour son salut. Pour accéder au sacerdoce, il faut donc y être spécialement appelé de Dieu ${ }^{19}$ ".

19. Dictionnaire de théologie catholique, article Vocation, col. 3149. 
Par ailleurs, une évolution se fait rapidement sentir. Il n'est plus question du sacerdoce en soi, mais de participation au sacerdoce du Christ; la description de la vocation divine est mise en relation avec la pastorale des vocations : l'action en faveur des vocations relève de la communauté chrétienne toute entière qui s'en acquitte d'abord par une vie pleinement chrétienne. En fait, la théologie de la vocation apparaît comme essentiellement tributaire de la théologie du sacerdoce et, déjà, d'une conception élargie et mieux située de la vocation baptismale.

\section{La doctrine conciliaire sur la vocation}

Ce qui se dégage en définitive de l'ensemble des textes conciliaires sur la vocation est d'abord une reprise et une mise au point des acquis antérieurs. La vocation sacerdotale correspond à une action particulière de Dieu. Ceci est indiqué par la reprise des notions de "vocation divine " et de "germe de vocation ". Elle implique corrélativement, une prise de conscience perçue comme un "appel " divin. La conception traditionnelle de la formation dans les petits séminaires sera d'ailleurs conservée. Cependant le Concile a explicitement abandonné l'expression "signes de vocation " de même que toute référence à la prédestination dont le concept est totalement éliminé.

La doctrine sur la vocation se situe enfin dans des perspectives nouvelles dont la prédominance d'une théologie historique. Le concept fondamental est celui d'histoire du salut présent dans les trois Constitutions sur l'Église, la Révélation, et l'Église dans le monde de ce temps. Le Concile se fonde sur la révélation de Dieu par son action dans l'histoire depuis la création du monde jusqu'à son achèvement dans le Christ. Cette perspective entraîne un élargissement de la notion de vocation. D'abord en ce qui concerne la vocation à la vie divine et la création. Bien au-delà du sens subjectif et restreint d'attrait vers la vie religieuse ou sacerdotale, le Concile remet en valeur l'initiative divine pour tous. Cette vocation à entrer dans la vie divine apparaît comme la structure spirituelle de la création elle-même. Par le fait même, la vocation chrétienne intégrale, en elle-même et dans ses diverses déterminations, telles que la vocation à la vie laïque, au mariage, au sacerdoce ou à la vie selon les conseils évangéliques est traitée de manière complète dans la Constitution Lumen gentium sur l'Église, dont le chapitre cinq sur l'appel universel à la sainteté développe cette requête à partir du baptême. Cette initiative divine qui fait entrer chaque chrétien dans le plan salvifique de Dieu est l'origine d'une vocation à la sainteté, antérieure à toute différenciation de vocation particulière. Ces divers éléments permettent une vue globale de la vocation comme concept fondamental pour exprimer la relation de l'homme à Dieu. 


\section{Les éléments constitutifs de la vocation}

Le primat de l'appel de Dieu comme révélation de son amour fait que la vocation est universelle. Tous sont appelés dans le Christ dès avant la création du monde et c'est la signification de cette découverte de l'amour de Dieu dans notre vie que nous appelons une vocation, qui consiste donc dans l'accueil conscient de cette révélation. La vocation implique en outre la certitude. L'action de l'Esprit Saint donne l'expérience de la foi qui n'est pas d'abord un ensemble de "vérités à croire ", mais adhésion à une personne, un don, un engagement. Ainsi, la vocation dans son sens plénier inclut le mystère de la rencontre personnelle avec Dieu. Il peut y avoir des infidélités, mais on ne peut pas perdre sa vocation car il y a toujours et pour tous à tous les moments de la vie la fidélité de Dieu à ses promesses.

En conclusion, les notations historiques recueillies ci-dessus et l'enseignement conciliaire peuvent ouvrir à quelques réflexions

1- Sur l'appel de Dieu. L'idée de vocation paraît claire et aller de soi : Dieu appelle! L'expression prend certes tout son sens quand elle se réfère au Dieu qui agit et parle dans l'Écriture, et qui se révèle pleinement en Jésus-Christ. Encore faut-il percevoir cet appel et percevoir qu'il vient bien de Dieu. Mais, alors que l'Écriture Sainte montre à travers l'histoire du peuple élu et les inspirations prophétiques une lente " conversion " de la pensée humaine dans son appréhension de la relation de l'homme à Dieu, l'histoire de l'Église fait découvrir que souvent la pureté de l'appel évangélique a été contaminée par le retour à des idées sur Dieu inspirées surtout par une religiosité spontanée, qui absolutise en Dieu des concepts issus de l'expérience humaine, tels que ceux de pouvoir, de puissance, de science, de justice, etc. Et ce ne sont pas seulement des expressions du vocabulaire religieux qui ont souffert de ce que le $P$. Sesboüé a nommé un phénomène de " déconversion ${ }^{20}$ ", mais aussi des termes issus de la tradition philosophique. Or, l'idée de vocation, est en étroite dépendance de la conception de Dieu qui inspire la réflexion. S'il s'agit d'une conception contaminée par un déisme rationaliste, l'idée de vocation conçue de manière conceptuelle comme pensée éternelle de Dieu se trouve nécessairement liée à celle de prédestination avec tout ce que cela entraîne d'erreurs, y compris dans la formation. Sans doute, historiquement, depuis le renouveau après la Révolution, cette liaison est restée longtemps, nous l'avons noté, à l'arrière plan, contestée au fond par le mouvement même de la vie chrétienne fondée sur la foi, comme le montre l'histoire des vocations sacerdotales et religieuses au cours des derniers siècles. On peut se demander cependant si ces fausses notions de Dieu, plus naturelles que chrétiennes, n'ont pas contribué à pervertir le sens de la vocation. Celle-ci n'a de sens, en effet, que dans la révélation du Christ.

20. SESBoüÉ, Bernard, Jésus-Christ l'unique médiateur. Essai sur la rédemption et le salut. Paris, Desclée, 1988, tome 1, p. 59. 
2-Sur la réponse à l'appel. Que signifie la conscience d'être appelé? Certes, la vocation trouve sa certitude dans l'expérience spirituelle qu'elle inclut, expérience développée et éprouvée en Église. Cependant, en tant que réalité psychologique et sociale, la vocation entre aussi entièrement dans le champ des sciences humaines et sera donc toujours réductible en apparence à une perspective purement humaine; et il en sera de même de toutes les médiations familiales, ecclésiales, etc., qui entrent dans la pastorale des vocations et la formation des prêtres. Mais le concept même de vocation n'a de sens que dans une perspective qui est celle de "l'obéissance de la foi " (Ro 1/5). Le modèle est l'expérience des disciples : expérience unique d'un appel divin, mais cet appel est humain dans sa formulation et Saint Thomas fait remarquer justement qu'il inclut l'appel intérieur de l'Esprit Saint, c'està-dire la foi. Nous sommes donc renvoyés à l'expérience chrétienne et à ses différentes formes. L'expérience spirituelle porte en elle-même sa certitude. C'est à l'approfondissement d'une telle conscience que le Concile appelle quand il enseigne la vocation universelle à la sainteté dans la vie familiale et professionnelle. Il en est de même quand la " conscience d'être appelé " marque la vocation religieuse ou sacerdotale.

La condition humaine, en effet implique que l'expérience de la foi sera toujours réductible psychologiquement à autre chose qu'elle-même, mais elle n'en porte pas moins aussi en elle-même l'affirmation de sa vérité, non pas vérité abstraite, mais dans l'Esprit-Saint vérité existentielle de la relation au Christ selon la parole de saint Paul : scio cui credidi « Je sais en qui j'ai mis ma foi » (2 Tim 1/12). 
RÉSUMÉ

En 1896, le chanoine Branchereau, supérieur du grand séminaire d'Orléans, publiait un livre De la vocation sacerdotale qui apparaissait comme une synthèse de la position courante qui avait inspiré au cours du siècle les efforts de renouveau des séminaires : la " vocation " est essentiellement une réalité intérieure, un appel divin qui se manifeste par des signes qu'il importe de discerner. Cependant, en 1909, le chanoine Lahitton, professeur au grand séminaire de Dax, publiait lui aussi un livre La vocation sacerdotale. Traité théorique et pratique dont la thèse était diamétralement opposée à la précédente : la vocation vient de Dieu par l'évêque. Il n'y a pas d'appel divin à chercher dans le sujet.

L'origine de la théologie de l'attrait au sens moderne doit être cherchée à la Renaissance et au $\mathrm{XVII}^{\mathrm{e}}$ siècle. Du côté catholique, on lutte contre les ordinations de prêtres faites pour des raisons financières et de situation sociale; du côté des Réformateurs, Luther et Calvin, on insiste sur la responsabilité personnelle de chacun, sa " vocation ", face à un appel de Dieu adressé à tous quel que soit son métier ou sa situation sociale.

Peu à peu émergea dans le monde catholique l'idée de vocation sacerdotale comme " vocation divine " qui conduisit à une théologie de l'attrait et, chez certains, à celle d'une vocation conçue commme une prédestination. Une telle conception influa grandement sur la conception des vocations d'enfants et leur recrutement.

La querelle des théologiens entre deux conceptions aussi incompatibles fut vive et connut des épisodes multiples : approbation de la thèse de Lahitton par une commission cardinalice en 1912 et plus tard diverses mises au point par les papes Pie XI et Pie XII. Le Concile Vatican II opéra une synthèse d'abord dans la "Constitution sur l'Église " par sa conception de la vocation universelle à la sainteté, antérieure à toute différentiation selon des vocations particulières, puis par une conception plus élaborée du sacerdoce ministériel comme participation à l'unique sacerdoce du Christ.

\section{ABSTRACT}

In 1896, Canon Branchereau, who was Superior of the Major Seminary at Orléans, published About the priestly vocation, a book which seemed to be a synthesis of the current position, which had inspired the seminary revival efforts all along the century: a vocation is essentially an inner reality, a divine call which occurs through signs to be discerned.

However, in 1909, Canon Lahitton, professor at the Dax Major Seminary also published a book: The Priestly Vocation. A Theoretical and Practical Treatise. His the sis was diametrically opposed to the first one: a vocation comes from God via the bishop; there is no divine call to be searched for in the subjet; the origin of the modern "attraction theology" are seated "théologie de l'attrait" has to be searched in the Renaissance and in the $17^{\text {th }}$ Century. On the catholic side, it is the fight against priestly ordinations motivated by financial or social reasons; on the Reformation side, Luther and Calvin, it was the insistance on the responsability of each individual candidate, his own vocation, as opposed to God's call directed to everybody, for whatever work and in whatever social position.

Step by step, in the catholic world, the priestly vocation emerged as "the divine vocation"; this led, in turn, to the theology of attraction, and even to the notion of a predestined vocation. Such a conception greatly influenced the vocation of children and their recruitment as seminarians. The theological dispute between two such incompatible conceptions was sharp and knew many events: Lahitton's thesis was approved by a cardinalice commission in 1912, and later adjusted by Popes Pius XI and Pius XII. Vatican Concil II proposeded a synthesis of both, first in the "Constitution on the Church" by its concept of universal vocation to holiness, before any differentiation by particular vocations; and later a more elaborated conception of the ministerial Priesthood as participation to Christ's priesthood. 